\title{
Spectroscopic Discovery of the Supernova 2003dh Associated with GRB 030329 ${ }^{1}$
}

\author{
K. Z. Stanek ${ }^{2}$, T. Matheson ${ }^{2}$, P. M. Garnavich ${ }^{3}$, P. Martini ${ }^{4}$, P. Berlind ${ }^{5}$, N. Caldwell ${ }^{2}$,

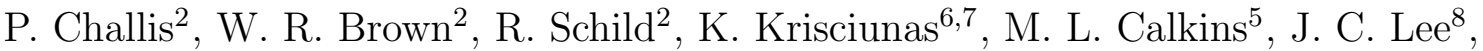 \\ N. Hathi ${ }^{9}$, R. A. Jansen ${ }^{9}$, R. Windhorst ${ }^{9}$, L. Echevarria ${ }^{9}$, D. J. Eisenstein ${ }^{8}$, B. Pindor ${ }^{10}$, \\ E. W. Olszewski ${ }^{8}$, P. Harding ${ }^{11}$, S. T. Holland ${ }^{3}$, D. Bersier ${ }^{2}$
}

\begin{abstract}
We present early observations of the afterglow of the Gamma-Ray Burst (GRB) 030329 and the spectroscopic discovery of its associated supernova SN 2003dh. We obtained spectra of the afterglow of GRB 030329 each night from March 30.12 (0.6 days after the burst) to April 8.13 (UT) (9.6 days after the burst). The spectra cover a wavelength range of $350 \mathrm{~nm}$ to $850 \mathrm{~nm}$. The early spectra consist of a power-law continuum $\left(F_{\nu} \propto \nu^{-0.9}\right)$ with narrow emission lines originating from HII regions in the host galaxy, indicating a low redshift of
\end{abstract}

\footnotetext{
${ }^{1}$ Based on data from the MMTO 6.5m telescope, the Magellan 6.5m Clay telescope, and the FLWO 1.5m telescope

${ }^{2}$ Harvard-Smithsonian Center for Astrophysics, 60 Garden Street, Cambridge, MA 02138; kstanek, tmatheson, ncaldwell, pchallis, wbrown, rschild, dbersier@cfa.harvard.edu

${ }^{3}$ Department of Physics, University of Notre Dame, 225 Nieuwland Science Hall, Notre Dame, IN 46556; pgarnavi@miranda.phys.nd.edu, sholland@nd.edu

${ }^{4}$ Carnegie Observatories, 813 Santa Barbara Street, Pasadena, CA 91101; martini@ociw.edu

${ }^{5}$ Smithsonian Institution, F. L. Whipple Observatory, 670 Mt. Hopkins Road, P.O. Box 97, Amado, AZ 85645; pberlind, mcalkins@cfa.harvard.edu

${ }^{6}$ Las Campanas Observatory, Casilla 601, La Serena, Chile

${ }^{7}$ Cerro Tololo Inter-American Observatory, Casilla 603, La Serena, Chile; kevin@ctiosz.ctio.noao.edu

${ }^{8}$ Steward Observatory, University of Arizona, 933 N. Cherry Ave., Tucson, AZ 85718; janice@pompelmo.as.arizona.edu, eisenste@cmb.as.arizona.edu, edo@adansonia.as.arizona.edu

${ }^{9}$ Department of Physics and Astronomy, Arizona State University, Tempe, AZ 85287-1504; Nimish, Rolf.Jansen, Rogier.Windhorst, Luis.Echevarria@asu.edu

${ }^{10}$ Princeton University Observatory, Princeton, NJ 08544; pindor@astro.princeton.edu

${ }^{11}$ Department of Astronomy, Case Western Reserve University, 10900 Euclid Avenue, Cleveland, OH 44106; harding@billabong.astr.cwru.edu
} 
$z=0.1687$. However, our spectra taken after 2003 Apr. 5 show broad peaks in flux characteristic of a supernova. Correcting for the afterglow emission, we find the spectrum of the supernova is remarkably similar to the type Ic 'hypernova' SN 1998bw. While the presence of supernovae have been inferred from the light curves and colors of GRB afterglows in the past, this is the first direct, spectroscopic confirmation that a subset of classical gamma-ray bursts originate from supernovae.

Subject headings: galaxies: distances and redshifts - gamma-rays: bursts supernovae: general — supernovae: individual (SN 2003dh)

\section{Introduction}

The origin of gamma-ray bursts (GRB) has been a mystery since their discovery in the 1960's. It has only been since the BeppoSAX satellite (Boella et al. 1997) began providing rapid, accurate localization of several bursts per year that it has it been possible to study these events and their afterglows in detail. Optical observations of afterglows (e.g. GRB 970228: Groot et al. 1997; van Paradijs et al. 1997) have allowed redshifts to be measured for a number of GRBs (e.g. GRB 970508: Metzger et al. 1997), providing definitive proof of their cosmological origin. GRB 980425 was likely associated with supernova 1998bw and this was the first direct evidence that at least some GRB result from the core collapse of massive stars (Galama et al. 1998). However the isotropic energy of that burst was $10^{-3}$ to $10^{-4}$ times weaker (Woosley, Eastman, \& Schmidt 1999) than classical cosmological GRB which placed it in a unique class. Indirect evidence of the connection between GRB and massive stars has come from studies of the location of GRB in their host galaxies (e.g. Holland \& Hjorth 1999) and statistics on the types of galaxies that host GRB (e.g. Hogg \& Fruchter 1999). Chevalier \& Li (2000) have shown that the afterglow properties of some GRB are consistent with a shock moving into a stellar wind formed from a massive star.

Direct evidence of a classical GRB/supernova connection has been difficult to obtain because the typical redshift of a GRB is $z \sim 1$, meaning even powerful supernovae would peak at $R>23$ mag. A number of GRB have shown late deviations from a power-law decline (e.g. GRB 980326, Bloom et al. 1999) which are suggestive of a supernova peaking a few weeks after the burst. At $z=0.36$, GRB 011121/SN2001ke was a relatively nearby burst which showed a late-time bump and color changes consistent with a supernova (Garnavich et al. 2003a; Bloom et al. 2002). That burst was indeed the best evidence to date that classical, long gamma-ray bursts are generated by core-collapse supernovae, but it lacked a clear spectroscopic detection of a supernova signature. Detection of such a signature is 
reported in this paper for the GRB 030329.

The extremely bright GRB 030329 was detected by the FREGATE, WXM, SXC instruments aboard HETE II at 11:14:14.67 UT on 2003 March 29 (Vanderspek et al. 2003). The burst falls in the 'long' category with a duration of $>25$ seconds. Peterson \& Price (2003) and Torii (2003) reported discovery of a very bright $(R \sim 13)$, slowly fading (Uemura 2003) optical transient (OT), located at $\alpha=10^{\mathrm{h}} 44^{\mathrm{m}} 50.0, \delta=+21^{\circ} 31^{\prime} 17^{\prime \prime} .8$ (J2000.0), and identified this as the GRB optical afterglow. Due to the brightness of the afterglow and its slow decay, photometric observations were extensive, making it one of the best-observed afterglows.

The afterglow was also very bright in X-rays (Marshall \& Swank 2003), radio (Berger, Soderberg \& Frail 2003), sub-millimeter (Hoge et al. 2003) and infrared (Lamb et al. 2003). Using archival data Blake \& Bloom (2003) have put a $3 \sigma$ upper limit of $\mathrm{R}=22.5$ on the brightness of the host. Martini et al. (2003) were the first to report optical spectroscopy of the afterglow. Because the very bright OT overwhelmed the emission from the faint host galaxy, only a single emission line was detected and confirmed by Della Ceca et al. (2003). Suggesting that this line may be due to [OII] provided a redshift of $z \approx 0.5$. However, a high-resolution VLT spectrum by Greiner et al. (2003) revealed additional emission and also absorption lines which fixed the redshift at a very low $z=0.1685$. This was later confirmed by Caldwell et al. (2003), making GRB 030329 the second nearest burst overall (GRB 980425 is the nearest at $\mathrm{z}=0.0085)$ and the classical burst with the lowest known redshift.

\section{Observations}

From the moment the low redshift for the GRB 030329 was announced (Greiner et al. 2003), we started organizing a campaign of spectroscopic and photometric follow-up of the afterglow and later the possible associated supernova. Spectra of the OT associated with GRB 030329 were obtained over many nights with the $6.5 \mathrm{~m}$ MMT telescope, the $1.5 \mathrm{~m}$ Tillinghast telescope at the F. L. Whipple Observatory (FLWO), and the Magellan 6.5m Clay telescope. The spectrographs used were the Blue Channel (Schmidt et al. 1989) at the MMT, FAST (Fabricant et al. 1998) at the FLWO 60" telescope, and LDSS2 (Mulchaey 2001) at Magellan. The observations were reduced in the standard manner with $\mathrm{IRAF}^{12}$ and our own routines. Spectra were optimally extracted (Horne 1986). Wavelength calibration was accomplished with HeNeAr lamps taken immediately after each OT exposure. Small-scale

\footnotetext{
${ }^{12}$ IRAF is distributed by the National Optical Astronomy Observatory, which is operated by the Association of Universities for Research in Astronomy, Inc., under cooperative agreement with the National Science Foundation.
} 
adjustments derived from night-sky lines in the OT frames were also applied. We used Feige 34 (Stone 1977) and HD 84937 (Oke \& Gunn 1983) as spectrophotometric standards. We attempted to remove telluric lines using the well-exposed continua of the spectrophotometric standards (Wade \& Horne 1988; Matheson et al. 2000). The spectra were in general taken at or near the parallactic angle (Filippenko 1982). The relative fluxes are thus accurate to $\sim 5 \%$ over the entire wavelength range. Figure 1 shows a subset of our spectra. The initial report on some of these data was presented by Martini et al. (2003) and Caldwell et al. (2003). Circulars by Matheson et al. (2003a), Garnavich et al. (2003b), Matheson et al. (2003b) and Garnavich et al. (2003c) reported the first detection of supernova associated with GRB 030329 (see next section).

\section{Results}

The brightness of the OT and its slow and uneven rate of decline, with episodes of increased brightness (well documented by many GCN circulars) allowed us to observe the OT every night since the GRB event (so far until Apr. 9 UT), thus providing a unique opportunity to look for spectroscopic evolution over many nights. The early spectra of the OT of GRB 030329 (top of Figure 1) consist of a power-law continuum typical of GRB afterglows, with narrow emission features identifiable as $\mathrm{H} \alpha$, [OIII], $\mathrm{H} \beta$ and [OII] at $z=$ 0.1687 (Greiner et al. 2003; Caldwell et al. 2003) probably from HII regions in the host galaxy. These lines can be used to estimate the star formation rate within the spectrograph slit (Kennicutt 1998). Preliminary analysis of the [OII] flux suggests a low star formation rate of $\sim 0.1 M_{\odot} y r^{-1}$ (Caldwell et al. 2003), but a more detailed analysis is possible after the afterglow has faded.

A fit to the early spectra provides a power-law index of $\beta=-0.94+/-0.01$ for April 1 and $-0.93+/-0.01$ for April 4 (statistical errors only). This is consistent with the spectral slope of -0.94 found using SDSS photometry taken March 31/April 1 (Lee et al. 2003). Correcting for low Galactic extinction of $E(B-V)=0.025$ (Schlegel et al. 1998) lowers the slope to -0.85 which is a typical spectral index for GRB afterglows (e.g. Stanek et al. 2001).

Beginning April 6, our spectra showed the development of broad peaks in flux, characteristic of a supernova. The broad bumps are seen at approximately $5000 \AA$ and $4200 \AA$ (rest frame). At that time, the spectrum of GRB 030329 looked similar to that of the peculiar type Ic SN 1998bw a week before maximum light (Patat et al. 2001). Over the next few days the SN features became more prominent as the afterglow faded and the SN brightened towards maximum. 
To discern the spectrum of the SN component, we assume that the spectral slope of the afterglow light did not evolve significantly in time. This allows us to subtract the afterglow dominated spectrum obtained on April 4 UT (see Figure 1) from the supernova dominated spectrum of April 8. The resulting spectrum is shown in Fig 2. For comparison, spectra of SN 1998bw at maximum and a week before maximum are also shown (Patat et al. 2001). The similarities of the GRB 030329 supernova to SN 1998bw are striking while the match to other 'hypernovae' such as SN 1997ef (Iwamoto et al. 2000) and SN 2002ap (Mazzali et al. 2002) is not as good. The primary difference is that the feature around $4400 \AA$ (rest frame) in GRB 030329 supernova and SN 1998bw is very broad while in SN 1997ef and SN 2002ap the feature is sharp and well defined. This is likely an indication that the expansion velocities in 1998bw and GRB 030329 supernova are significantly higher than in the other two events.

The evolution of the GRB 030329/SN 2003dh is still ongoing. In a future paper (Matheson et al. 2003, in preparation) we will discuss in more detail the properties of the afterglow of GRB 030329, in itself a very unusual event, and long-term spectroscopic and photometric evolution of the SN 2003dh. With this future paper we will also release our data for this GRB/SN via anonymous ftp.

We have shown convincing spectroscopic evidence that a supernova was lurking beneath the optical afterglow of the classical, long gamma-ray burst 030329. The supernova spectrum was very similar to the type Ic 'hypernova' SN 1998bw which was associated with the intrinsically weak GRB 980425. With two confirmed cases, it is tempting to link only type Ib/c events with GRB. However, the type IIn hypernova 1997cy (Germany et al. 2000) may have triggered a GRB and the blue color of SN 2001ke near maximum was consistent with some hydrogen rich events (Garnavich et al. 2003a).

The $4400 \AA$ feature in the spectrum of GRB 030329 is significantly broader than seen in 'hypernovae' SN 1997ef and SN 2002ap, neither of which was clearly associated with a GRB event. While the presence of supernovae have been inferred from the light curves and colors of GRB afterglows in the past, this is the first direct, spectroscopic confirmation that some and maybe all classical gamma-ray bursts originate from supernovae.

We would like to thank the staffs of the MMT, Las Campanas and the FLWO observatories. Thanks to A. Milone for helping with the MMT spectra. PMG acknowledges the support of NASA/LTSA grant NAG5-9364. PM was supported by a Carnegie Starr Fellowship. JCL acknowledges financial support from NSF grant AST-9900789. DJE was supported by NSF grant AST-0098577 and by an Alfred P. Sloan Research Fellowship. BP is supported by NASA grant NAG5-9274. EWO was partially supported by NSF grant AST 0098518. 


\section{REFERENCES}

Berger, E., Soderberg, A. M., \& Frail, D. A. 2003, GCN Circ. 2014

Blake, C., \& Bloom, J. S. 2003, GCN Circ. 2011

Bloom, J. S., et al. 1999, Nature, 401, 453

Bloom, J. S., et al. 2002, ApJ, 572, L45

Boella, G., Butler, R. C., Perola, G. C., Piro, L., Scarsi, L., \& Bleeker, J. A. M. 1997, A\&AS, 122,299

Caldwell, N., Garnavich, P., Holland, S., Matheson, T., \& Stanek, K.Z. 2003, GCN Circ. 2053

Chevalier, R.A., \& Li, Z. 2000, ApJ, 520, 29

Della Ceca, R., Maccacaro, T., Fugazza, D., Pedani, M., Cecconi, M., Fiore, F., Antonelli, L.A., Covino, S., Pian, E.,\& Masetti, N. 2003, GCN Circ. 2015

Fabricant, D., Cheimets, P., Caldwell, N., \& Geary, J. 1998, PASP, 110, 79

Filippenko, A. V. 1982, PASP, 94, 715

Galama, T. J., et al. 1998, ApJ, 497, L13

Garnavich, P. M., et al. 2003a, ApJ, 582, 924

Garnavich, P., Matheson, T., Eisenstein, D., Pindor, B., Hathi, N., Jansen, R., Windhorst, R., Echevarria, L., Lee, J., Krisciunas, K., Martini, P., Brown, W., Caldwell, N., Berlind, P., Calkins, M., \& Stanek, K. Z. 2003c, IAU Circ. 8108

Garnavich, P., Matheson, T., Olszewski, E. W., Harding, P., \& Stanek, K. Z. 2003c, IAU Circ. 8114

Germany, L. M., Reiss, D. J., Sadler, E. M., Schmidt, B. P., \& Stubbs, C. W. 2000, ApJ, 533,320

Greiner, J., et al. 2003, GCN Circ. 2020

Groot, P. J. et al. 1997, IAU Circ. 6584

Hoge, J. C., Meijerink, R., Tilanus, R. P. J., \& Smith, I. A. 2003, GCN Circ. 2088

Hogg, D. W., \& Fruchter, A. S. 1999, ApJ, 520, 54 
Holland, S. T., \& Hjorth, J. 1999, A\&A, 344, L67

Horne, K. 1986, PASP, 98, 609

Iwamoto, K., Nakamura, T., Nomoto, K., Mazzali, P. A., Danziger, I. J., Garnavich, P., Kirshner, R., Jha, S., Balam, D., \& Thorstensen, J. 2000, ApJ, 534, 660

Kennicutt, R.C 1998, ARAA, 36, 189

Lamb, D.Q., York, D., Barentine, J., Ketzebeck, B., Dembicky, J., McMillan, R., Nysewander, M., \& Reichart, D. 2003, GCN Circ. 2040

Lee, B.C., Lamb, D.Q., Tucker, D.L., \& Kent, S. 2003, GCN Circ. 2096

Martini, P., Garnavich, P. M., \& Stanek, K. Z. 2003, GCN Circ. 2013

Marshall, F.E, \& Swank, J.H. 2003, GCN Circ. 1996

Matheson, T., Filippenko, A. V., Ho, L. C., Barth, A. J., \& Leonard, D. C. 2000, AJ, 120, 1499

Matheson, T., Garnavich, P., Hathi, N., Jansen, R., Windhorst, R., Echevarria, L., Lee, J., Brown, W., Caldwell, N., Berlind, P., Calkins, M., \& K. Z. Stanek 2003a, GCN Circ. 2107

Matheson, T., Garnavich, Olszewski, E.W., Harding, P., Eisenstein, D., Pindor, B., P., Hathi, N., Jansen, R., Windhorst, R., Echevarria, L., Lee, J., Brown, W., Caldwell, N., Berlind, P., Calkins, M., \& K. Z. Stanek 2003b, GCN Circ. 2120

Mazzali, P. A., Deng, J., Maeda, K., et al. 2002, ApJ, 572, L61

Metzger, M. R., Djorgovski, S. G., Kulkarni, S. R., Steidel, C. C., Adelberger, K. L., Frail, D. A., Costa, E., \& Frontera, F. 1997, Nature, 387, 878

Mulchaey, J. 2001, http://www.ociw.edu/magellan_lco/instruments/LDSS2/

Oke, J. B., \& Gunn J. E. 1983, ApJ, 266, 713

Patat, F., Cappellaro, E., Danziger, J., et al. 2001, ApJ, 555, 900

Peterson, B. A., \& Price, P. A. 2003, GCN Circ. 1985

Schlegel, D. J., Finkbeiner, D. P., \& Davis, M. 1998, ApJ, 500, 525

Schmidt, G., Weymann, R. \& Foltz, C. 1989, PASP, 101, 713 
Stanek, K. Z., Garnavich, P. M., Jha, S., Kilgard, R. E., McDowell, J. C., Bersier, D., Challis, P., Falco, E., \& Quinn, J. L. 2001, ApJ, 554, 155

Stone, R. P. S. 1977, ApJ, 218, 767

Torii, K. 2003, GCN Circ. 1986

Uemura, M. 2003, GCN Circ. 1989

Vanderspek, R., et al. 2003, GCN Circ. 1997

van Paradijs, J., et al. 1997, Nature, 386, 686

Wade, R. A., \& Horne, K. D. 1988, ApJ, 324, 411

Woosley, S. E., Eastman, R. G., \& Schmidt, B. P. 1999, ApJ, 516, 788 


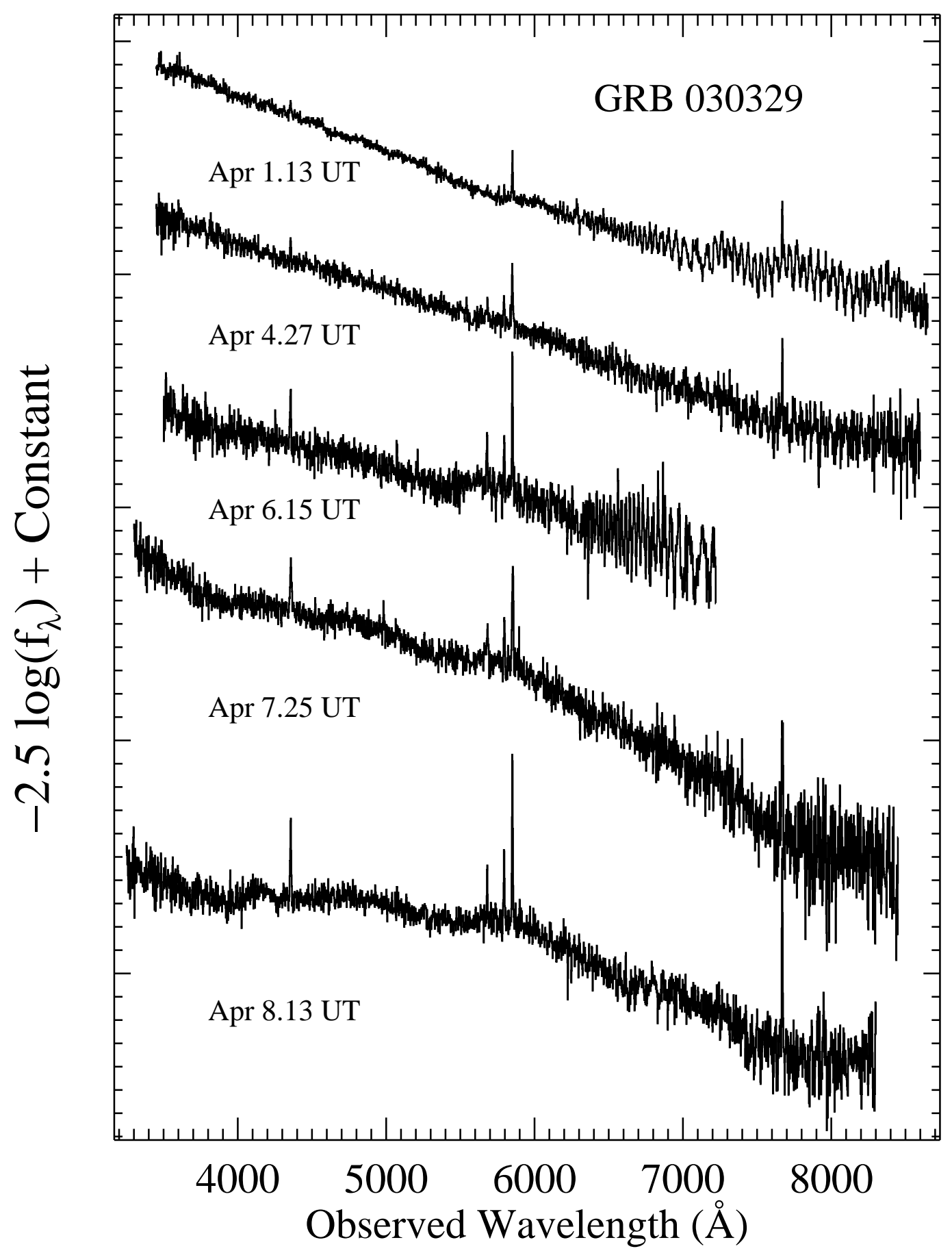

Fig. 1.- Evolution of the GRB 03029/SN 2003 spectrum, from April 1.13 UT (2.64 days after the burst), to April 8.13 UT (9.64 days after the burst). The early spectra consist of a power-law continuum $\left(F_{\nu} \propto \nu^{-0.9}\right)$ with narrow emission lines originating from HII regions in the host galaxy at a redshift of $\mathrm{z}=0.168$. Spectra taken after Apr. 5 show the development of broad peaks in the spectra characteristic of a supernova. 


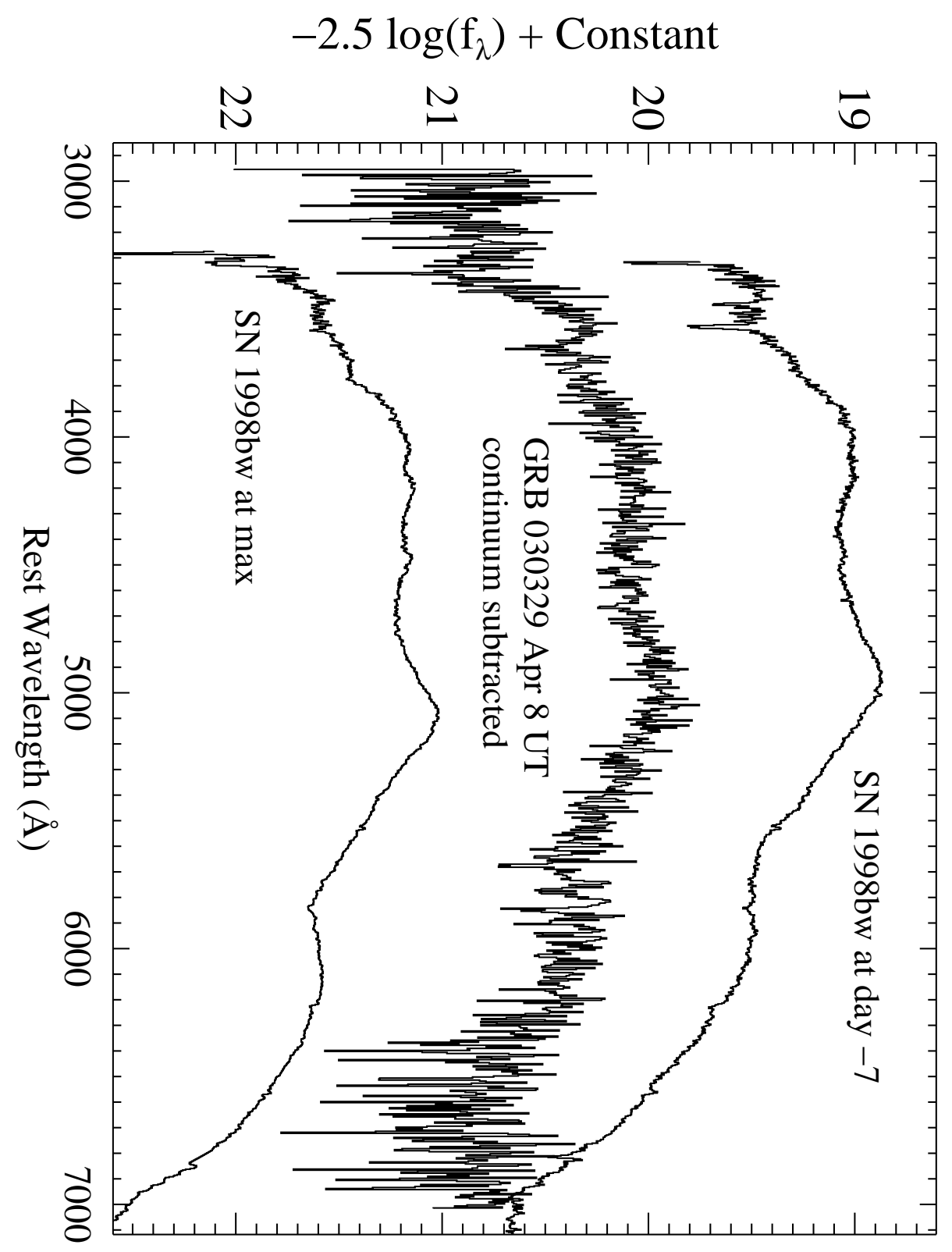

Fig. 2.- MMT spectrum of April 8 with the smoothed MMT spectrum of April 1 scaled and subtracted. The residual spectrum shows broad bumps at approximately $5000 \AA$ and $4200 \AA$ (rest frame), which is similar to the spectrum of the peculiar type Ic SN 1998bw a week before maximum light (Patat et al. 2001). The match is not as good for SN 1998bw at maximum light, especially at the red end of the spectrum. 